\title{
BDNF Serum Concentrations in Healthy Volunteers are Associated with Depression-Related Personality Traits
}

\author{
Undine E Lang',*, Rainer Hellweg' and Jürgen Gallinat ${ }^{2}$ \\ 'Clinic for Psychiatry and Psychotherapy, Charité Medicine Berlin, Campus Benjamin Franklin, Germany; ${ }^{2}$ Clinic for Psychiatry and Psychotherapy, \\ Charité Medicine Berlin, Campus Charité Mitte, Germany
}

\begin{abstract}
The issue of neurotrophins is recognized as a new lead in the quest for a deeper understanding of mood disorders. This hypothesis has emerged from experimental evidence suggesting that antidepressant drugs might work by a neuroprotective effect through the stimulation of the neurotrophin expression in distinct regions of the CNS. Endogenous levels of BDNF protein were measured in the serum samples of I I 8 healthy unrelated volunteers ( 64 male, 54 female, age: $42.1 \pm 13.0$ years), and the NEO-FFI has been performed in all subjects. BDNF serum values amounted to $16.3 \pm 7.3 \mathrm{ng} / \mathrm{ml}$. BDNF concentration correlated significantly with age $(r=0.182$, $p=0.048$ ), but showed no gender differences (male $16.1 \pm 7.2$, female $16.5 \pm 7.4 \mathrm{ng} / \mathrm{ml}$ ). A negative correlation between the BDNF serum concentration and the depression-related factor neuroticism $(r=-0.212, p=0.022)$ has been found. Low BDNF levels in healthy humans with depressive personality traits might constitute a risk marker, reflecting a personality profilethat is linked to vulnerability to mood disorders. These results provide further support for the hypothesis that BDNF may be central to the development of depressive mood states.

Neuropsychopharmacology (2004) 29, 795-798, advance online publication, 2 I January 2004; doi: I 0. I 038/sj.npp. 1300382
\end{abstract}

Keywords: depression; brain-derived neurotrophic factor; healthy volunteers; neuroticism; NEO-five factor inventory; personality

\section{INTRODUCTION}

The NEO-five factor inventory (NEO-FFI) is a psychometrically sound and widely used instrument, which assesses five global personality domains and one of them constitutes neuroticism (Costa and McCrae, 1992). Neuroticism is a strong marker for vulnerability to depression (Duggan $e t$ al, 1995) and a personality trait accompanied by anxiety, low mood, and hostility. New experimental evidence suggests that mood disorders, which have traditionally been conceptualized as neurochemical disorders, are also associated with impairments of structural plasticity and cellular resilience (Manji et al, 2003). Based on clinical and animal studies, it has been suggested that mood disorders are associated with neuronal atrophy and neuronal cell loss (Duman et al, 2000), especially in the hippocampus (Sheline, 2000) and cerebral cortex (Rajkowska, 2000). Therefore, the issue of neuroprotection and neurotrophins is recognized as an important new lead in the quest for a deeper understanding of mood disorders and the mechan-

\footnotetext{
*Correspondence: Dr UE Lang, Department of Psychiatry, Charité Medicine Berlin, Campus Benjamin Franklin, Eschenallee 3, Berlin 14050, Germany, Tel: + 49-30-8445-8302, Fax: + 49-30-8445-8350, E-mail: undine.lang@medizin.fu-berlin.de

Received 22 October 2003; revised 02 December 2003; accepted I। December 2003

Online publication: 16 December 2003 at http://www.acnp.org/ citations/Npp | 2 1 60303484/default.pdf
}

isms of action of antidepressants and mood stabilizers (Duman et al, 1997; Altar, 1999). This hypothesis has emerged especially from recent experimental evidence suggesting that antidepressant drugs and electroconvulsive treatment might work by a neuroprotective effect through the enhancement of the neurotrophin expression in distinct regions of the CNS (Duman et al, 1997; Altar, 1999; Siuciak et al, 1997). Previous animal studies have shown that hippocampal BDNF mRNA levels are significantly increased after physical exercise (Russo-Neustadt et al, 2000), administered antidepressant medications, and electroconvulsive therapy (Duman et al, 2000, 1997; Altar, 1999; Russo-Neustadt et al, 2000, 2001; Duman and Vaidya, 1998; Nibuya et al, 1995; Russo-Neustadt, 2003). According to this hypothesis, the infusion of BDNF into the midbrain of depression-model rats has been followed by recovery of behavioral deficits (Siuciak et al, 1997; Shirayama et al, 2002). In humans, increased BDNF expression was found in dentate gyrus, hilus, and supragranular regions in subjects treated with antidepressant medications at the time of death, compared with antidepressant-untreated subjects (Chen et al, 2001). In humans, decreased serum BDNF levels were reported recently in major depressed patients (Karege et al, 2002a) and increasing BDNF concentrations have been observed after antidepressant treatment (Shimizu et al, 2003). These data suggest BDNF concentrations to be a trait connected with depressed mood, which is also in line with the recent finding that a BDNF coding variant is associated 
with a depression-related personality trait in the NEO-FFI (Sen et al, 2003). The question as to whether this depression-related trait is also correlated to BDNF protein serum concentrations in healthy never-treated human volunteers has been addressed in this study.

\section{SUBJECTS AND METHODS}

\section{Subjects}

A total of 118 healthy unrelated volunteers (64 male, 54 female, age: $42.1 \pm 13.0$ years) were recruited for this study in the Charite Medicine Berlin by newspaper advertisement. The study was approved by the ethics committee. The MiniInternational Neuropsychiatric Interview (Sheehan et al, 1998) was applied. Axis-I or axis-II disorders, axis-I diagnosis of first degree relatives, and psychotropic drug intake led to the exclusion of the study as detailed elsewhere (Gallinat et al, 2002). The NEO-FFI (Costa and McCrae, 1992) has been performed in all subjects, all of whom were unrelated individuals of German descent (Caucasians). The participants completed the German version of the NEO-FFI, which consists of 60 items and allows reliable and valid assessment of personality along the dimensions neuroticism, extraversion, openness to experiences, agreeableness, and conscientiousness (Borkenau and Ostendorf, 1993). After a complete description of the study to the subjects, written informed consent was obtained.

\section{Measurement of BDNF Levels}

Endogenous levels of BDNF were measured in the rethawed serum samples using commercial ELISA kits in principle according to the manufacturer's instructions (Promega Inc., Mannheim, Germany), but adapted to the fluorometric technique used also for nerve growth factor determination (Hellweg et al, 2003), and described in detail previously (Hellweg et al, 1989). The BDNF content was expressed as equivalents of recombinant human BDNF. The detection limit of the assay was $1 \mathrm{pg} / \mathrm{ml}$. Determinations of recovery, specific, and unspecific neurotrophin binding (the latter against mouse $\operatorname{IgG}_{1}$ obtained from MOPC 21) involved quadruplicate fluorescence determinations for each serum sample (Hellweg et al, 2003).

\section{Data Analysis}

The Kolmogorov-Smirnov test was employed to evaluate whether BDNF level is a normally distributed trait. Gender effects were tested using $T$-test for independent samples, and age effects were determined with Pearson's correlation test. BDNF was correlated with NEO scores using partial correlation test. The results are presented as means $\pm 1 \mathrm{SD}$. Analyses were carried out using statistical software (SPSS $\left.11.0^{\circledR}\right)$. A $p$-value of $p<0.05$ was considered significant, while $p<0.10$ was accepted in order to detect trends.

\section{RESULTS}

BDNF serum values in the healthy human population amounted to $16.3 \pm 7.3 \mathrm{ng} / \mathrm{ml}$, and the median amounted to $14.7 \mathrm{ng} / \mathrm{ml}$. The Kolmogorov-Smirnov test $(D=1.29$,

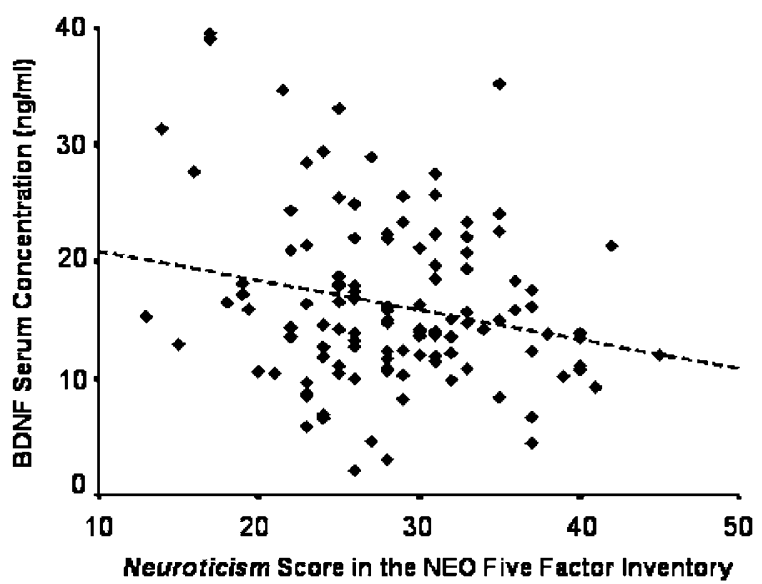

Figure I Correlation between brain-derived neurotrophic factor serum concentration $(\mathrm{ng} / \mathrm{ml})$ and neuroticism score in the NEO-FFI in healthy volunteers $(r=-0.212, N=|| 8, p=0.022)$

$p=0.07)$ showed that the BDNF serum concentrations in our sample were normally distributed. BDNF concentration correlated significantly with age $(r=0.182, p=0.048)$, but showed no gender differences (male 16.1 \pm 7.2 , female $16.5 \pm 7.4 \mathrm{ng} / \mathrm{ml} ; T=-0.343, \mathrm{df}=116, p=0.732 ; T$-test).

Significant age effects were found for the NEO factors neuroticism $(r=-0.218, p=0.018)$ and conscientiousness $(r=-0.246, p=0.007)$, but not for extraversion $(r=0.102$, $p=0.273)$, openness for experiences $(r=0.006, p=0.950)$, and agreeableness $(r=0.125, p=0.179)$. The effects of gender were observed in the factor neuroticism (male $26.9 \pm 5.9$, female $30.2 \pm 6.3 ; T=-2.927, \mathrm{df}=116, p=0.004)$ and agreeableness (male 42.5 \pm 5.5 , female $44.4 \pm 4.6$; $T=-2.039, \mathrm{df}=116, p=0.044)$, but not for extraversion (male $40.8 \pm 5.0$, female $40.9 \pm 6.4 ; T=-0.155, \mathrm{df}=116$, $p=0.877$ ), openness for experiences (male $42.3 \pm 6.0$, female $43.5 \pm 5.8 ; T=-1.105, \mathrm{df}=116, p=0.272)$, and conscientiousness (male 46.4 \pm 4.8 , female $46.0 \pm 5.5 ; T=0.381$, $\mathrm{df}=116, p=0.704)$.

Partial correlation test with the covariate age showed a significant negative correlation between the BDNF concentration and the factor neuroticism $(r=-0.212, p=0.022$; see Figure 1) and a positive correlation with the factor conscientiousness ( $r=0.225, p=0.015$ ).

This correlation has also been observed when age as a covariate has been removed for neuroticism $(r=-0.252$, $p=0.021)$ as for conscientiousness $(r=0.237, p=0.030)$.

No significant correlation between BDNF concentration and extraversion, openness, or agreeableness was observed $(r=0.115, p=0.217 ; r=0.032, p=0.728 ;$ and $r=0.109$, $p=0.142$, respectively).

\section{DISCUSSION}

This is, to our knowledge, the first study showing a negative correlation of BDNF serum concentrations and neuroticism in healthy volunteers. Low BDNF levels in healthy humans with depressive personality traits might reflect a genetic profile, which is linked to vulnerability to depression and therefore BDNF serum concentrations could serve as a risk marker. This point of view would be in line with the recent 
finding that also a BDNF coding variant is associated with the NEO personality inventory domain neuroticism (Sen et $a l, 2003)$. Also, BDNF gene association studies showed that the BDNF locus confers susceptibility to bipolar disorder (Neves-Pereira et al, 2002; Sklar et al, 2002). To verify this relationship, it also remains to be elucidated if serum BDNF levels are associated with the genetic polymorphisms examined. Higher BDNF serum values could therefore result from a genotype that might be protective against depression.

Another possibility to explain our results would be that BDNF serum concentrations are altered secondarily in a stress-dependent way. Stress can precipitate and exacerbate depression and may cause neuronal atrophy and death, especially in the hippocampus (McEwen, 2000; Sapolsky, 2000). Thus, a stress-induced BDNF reduction would inhibit the protective and trophic BDNF effects in the hippocampus. The reported antidepressant-induced neurogenesis in the hippocampus may be central to the antidepressive properties of antidepressant medications (Malberg et al, 2000), and this neurogenesis is possibly at least partly due to BDNF accumulation. Accordingly, a stress-induced reduction of hippocampal volumes (Sheline, 2000) and BDNF may be central to the development of depressive mood states (Rajkowska, 2000; Altar, 1999).

This hypothesis would be in line with data shown previously that physical exercise and antidepressant treatment increase the transcription of BDNF (Russo-Neustadt, 2003). Also, the infusion of BDNF into the midbrain of depression-model rats has been followed by recovery of behavioral deficits (Siuciak et al, 1997; Shirayama et al, 2002). In keeping with our current understanding of the cellular and molecular mechanisms of depression (Duman et al, 2000, 1997), monoaminergic neurotransmitter function may be an important component of these interventions. Accordingly, an augmentation of serotonergic activity within various brain areas following infusion of BDNF into the midbrain has been reported recently (Mossner et al, 2000). This serotonergic activity is due to the modulation of the serotonin transporter (Mossner et al, 2000).

One has to bear in mind, however, that BDNF serum changes in depressed subjects could be an epiphenomenona as the exact mechanisms of regulation of humoral BDNF levels are widely unknown. Platelets, brain neurons, and vascular endothelial cells are considered as candidate sources. A major source of the serum BDNF are platelets, which bind, store, and release BDNF upon activation and in response to coagulation stimuli (Yamamoto and Gurney, 1990; Fujimura et al, 2002). As platelets and neurons develop from a common embryonic precursor in the neural crest (Pearse, 1980), the peripheral BDNF concentration could possibly reflect the central neurotransmission state. A parallel BDNF brain and serum situation is underlined by the finding of Karege et al (2002b), who reported a positive correlation between the brain and serum BDNF levels in rats, which underwent similar changes during maturation and aging processes.

Previous studies on BDNF were mostly based on animal models, so an association of serum BDNF to the mood state in healthy human volunteers - as reported in this study- has not been shown yet. In contrast to previously reported observations (Chen et al, 2001; Karege et al, 2002a; Shimizu et al, 2003), an antidepressant drugassociated effect on BDNF could be ruled out in our sample of healthy human volunteers. These data suggest a causative relation between BDNF and mood states.

A decrease in serum BDNF levels might possibly influence the cascade relevant in the pathophysiology of depression also in the central nervous system, as it has been shown that neurotrophic factors from the blood stream can cross the blood-brain barrier under experimental conditions (Pan et al, 1998). However, there are also conflicting results showing that neurotrophins do not cross the blood-brain barrier (Pardridge, 2002). Therefore, it is premature to conclude that an upregulation of these factors in the blood will have beneficial effects in the brain. Whether such alterations are causatively associated with increased neural plasticity, improved cognitive function, and decreased depressive mood states remains to be elucidated in human studies.

\section{ACKNOWLEDGEMENTS}

We thank Heidi Danker-Hopfe for statistical advice. We gratefully acknowledge the excellent technical assistance of Mrs Bunge. This work was supported by the KFN, Kommission zur Förderung von Nachwuchswissenschaftlerinnen of the Free University of Berlin.

\section{REFERENCES}

Altar CA (1999). Neurotrophins and depression. Trends Pharmacol Sci 20: $59-61$.

Borkenau P, Ostendorf F (1993). NEO - Fünf - Faktoren Inventar (NEO-FFI), Handanweisung. Göttingen: Hogrefe.

Chen B, Dowlatshahi D, MacQueen GM, Wang JF, Young LT (2001). Increased hippocampal BDNF immunoreactivity in subjects treated with antidepressant medication. Biol Psychiatry 50: $260-265$.

Costa PT, McCrae RR (1992). Revised NEO Personality Inventory and NEO Five-Factor Inventory, Professional Manual, Psychological Assessment Resources: Odessa, FL.

Duggan C, Sham P, Lee A, Minne C, Murray R (1995). Neuroticism: a vulnerability marker for depression evidence from a family study. J Affect Disord 35: 139-143.

Duman RS, Heninger GR, Nestler EJ (1997). A molecular and cellular theory of depression. Arch Gen Psychiatry 54: 597-606.

Duman RS, Malberg J, Nakagawa S, D'Sa C (2000). Neuronal plasticity and survival in mood disorders. Biol Psychiatry 48: 732-739.

Duman RS, Vaidya VA (1998). Molecular and cellular actions of chronic electroconvulsive seizures. J ECT 14: 181-193.

Fujimura H, Altar CA, Chen R, Nakamura T, Nakahashi T, Kambayashi J et al (2002). Brain-derived neurotrophic factor is stored in human platelets and released by agonist stimulation. Thromb Haemost 87: 728-734.

Gallinat J, Mulert C, Bajbouj M, Herrmann WM, Schunter J, Senkowski D et al (2002). Frontal and temporal dysfunction of auditory stimulus processing in schizophrenia. Neuroimage 17: $110-127$.

Hellweg R, Hock C, Hartung HD (1989). An improved rapid and highly sensitive enzyme immunoassay for nerve growth factor. J Methods Cell Mol Biol 1: 43-49.

Hellweg R, von Armin CAF, Büchner M, Huber R, Riepe MW (2003). Neuroprotection and neuronal dysfunction upon repetitive disinhibition of oxidative phosphorylation. Exp Neurol 183: 346-354. 
Karege F, Perret G, Bondolfi G, Schwald M, Bertschy G, Aubry JM (2002a). Decreased serum brain-derived neurotrophic factor levels in major depressed patients. Psychiatry Res 109: 143-148.

Karege F, Schwald M, Cisse M (2002b). Postnatal developmental profile of brain-derived neurotrophic factor in rat brain and platelets. Neurosci Lett 328: 261-264.

Malberg JE, Eisch AJ, Nestler EJ, Duman RS (2000). Chronic antidepressant treatment increases neurogenesis in adult rat hippocampus. J Neurosci 20: 9104-9110.

Manji HK, Quiroz JA, Sporn J, Payne JL, Denicoff K, Gray NA et al (2003). Enhancing neuronal plasticity and cellular resilience to develop novel, improved therapeutics for difficult-to-treat depression. Biol Psychiatry 53: 707-742.

McEwen BS (2000). The neurobiology of stress: from serendipity to clinical relevance. Brain Res 886: 172-189.

Mossner R, Daniel S, Albert D, Heils A, Okladnova O, Schmitt A et al (2000). Serotonin transporter function is modulated by brain-derived neurotrophic factor (BDNF) but not nerve growth factor (NGF). Neurochem Int 36: 197-202.

Neves-Pereira M, Mundo E, Muglia P, King N, Macciardi F, Kennedy JL (2002). The brain-derived neurotrophic factor gene confers susceptibility to bipolar disorder: evidence from a family-based association study. Am J Hum Genet 71: 651-655.

Nibuya M, Morinobu S, Duman RS (1995). Regulation of BDNF and trkB mRNA in rat brain by chronic electroconvulsive seizure and antidepressant drug treatments. J Neurosci 15: 7539-7547.

Pan W, Banks WA, Fasold MB, Bluth J, Kastin AJ (1998). Transport of brain-derived neurotrophic factor across the blood-brain barrier. Neuropsychopharmacol 37: 1553-1561.

Pardridge WM (2002). Neurotrophins, neuroprotection and the blood-brain barrier. Curr Opin Invest Drugs 3: 1753-1757.

Pearse AG (1980). The common peptides and the cytochemistry of their cells of origin. Basic Appl Histochem 24: 63-73.

Rajkowska G (2000). Postmortem studies in mood disorders indicate altered numbers of neurons and glial cells. Biol Psychiatry 48: 766-777.

Russo-Neustadt A (2003). Brain-derived neurotrophic factor, behavior, and new directions for the treatment of mental disorders. Semin Clin Neuropsychiatry 8: 109-118.
Russo-Neustadt A, Ha T, Ramirez R, Kesslak JP (2001). Physical activity-antidepressant treatment combination: impact on brainderived neurotrophic factor and behavior in an animal model. Behav Brain Res 120: 87-95.

Russo-Neustadt AA, Beard RC, Huang YM, Cotman CW (2000). Physical activity and antidepressant treatment potentiate the expression of specific brain-derived neurotrophic factor transcripts in the rat hippocampus. Neuroscience 101: 305-312.

Sapolsky RM (2000). Glucocorticoids and hippocampal atrophy in neuropsychiatric disorders. Arch Gen Psychiatry 57: 925-935.

Sen S, Nesse RM, Stoltenberg SF, Li S, Gleiberman L, Chakravarti A et al (2003). A BDNF coding variant is associated with the NEO personality inventory domain neuroticism, a risk factor for depression. Neuropsychopharmacology 28: 397-401.

Sheehan DV, Lecrubier Y, Sheehan KH, Amorim P, Janavs J, Weiller E (1998). The Mini-International Neuropsychiatric Interview (M.I.N.I.): the development and validation of a structured diagnostic psychiatric interview for DSM-IV and ICD-10. J Clin Psychiatry 59: 22-33.

Sheline YI (2000). 3D MRI studies of neuroanatomic changes in unipolar major depression: the role of stress and medical comorbidity. Biol Psychiatry 48: 791-800.

Shimizu E, Hashimoto K, Okamura N, Koike K, Komatsu N, Kumakiri C et al (2003). Alterations of serum levels of brainderived neurotrophic factor (BDNF) in depressed patients with or without antidepressants. Biol Psychiatry 54: 70-75.

Shirayama Y, Chen AC, Nakagawa S, Russell DS, Duman RS (2002). Brain-derived neurotrophic factor produces antidepressant effects in behavioral models of depression. J Neurosci 22: 3251-3261.

Siuciak JA, Lewis DR, Wiegand SJ, Lindsay RM (1997). Antidepressant-like effect of brain-derived neurotrophic factor (BDNF). Pharmacol Biochem Behav 56: 131-137.

Sklar P, Gabriel SB, McInnis MG, Bennett P, Lim YM, Tsan G et al (2002). Family-based association study of 76 candidate genes in bipolar disorder: BDNF is a potential risk locus. Brain-derived neutrophic factor. Mol Psychiatry 7: 579-593.

Yamamoto H, Gurney ME (1990). Human platelets contain brainderived neurotrophic factor. J Neurosci 10: 3469-3478. 